\title{
Event-based sonification of EEG rhythms in real time
}

\author{
Gerold Baier ${ }^{\mathrm{a}, *}$, Thomas Hermann ${ }^{\mathrm{b}}$, Ulrich Stephani ${ }^{\mathrm{c}}$ \\ ${ }^{a}$ Facultad de Ciencias, Universidad Autonoma del Estado de Morelos, 62209 Cuernavaca, Mexico \\ ${ }^{\mathrm{b}}$ Neuroinformatics Group, Bielefeld University, 33615 Bielefeld, Germany \\ c Neuropediatric Clinic, University of Kiel, 24105 Kiel, Germany
}

Accepted 14 January 2007

Available online 29 March 2007

\begin{abstract}
Objective: To introduce a sound synthesis tool for human EEG rhythms that is applicable in real time.

Methods: We design an event-based sonification which suppresses irregular background and highlights normal and pathologic rhythmic activity.

Results: We generated sound examples with rhythms from well-known epileptic disorders and find stereotyped rhythmic auditory objects in single channel and stereo display from generalized spike-wave runs. For interictal activity, we were able to separate focal rhythms from background activity and thus enable the listener to perceive its frequency, duration, and intensity while monitoring.

Conclusions: The proposed event-based sonification allows quick detection and identification of different types of rhythmic EEE events in real time and can thus be used to complement visual displays in monitoring and EEG feedback tasks.

Significance: The significance of the work lies in the fact that it can be implemented for on-line monitoring of clinical EEG and for EEG feedback applications where continuous screen watching can be substituted or improved by the auditory information stream.

(C) 2007 International Federation of Clinical Neurophysiology. Published by Elsevier Ireland Ltd. All rights reserved.
\end{abstract}

Keywords: Electroencephalography; Epileptic rhythms; Sonification

\section{Introduction}

In EEG monitoring and EEG feedback quick detection and identification of rhythmic patterns in real time can be desirable. Typically, one watches the recordings displayed on a monitoring screen, i.e. a visual display. Auditory displays, in contrast, offer the advantage to follow the events without the need to watch a screen, even allowing observation while leaving the room. However, if recorded EEG is made audible directly by using the voltage level to drive a speaker membrane (audification), the result is mostly noisy. Such a display is both uncomfortable to listen to for long periods of time and it does not well support the discrimination of rhythmic features from their noisy background. \footnotetext{
40.

* Corresponding author. Tel.: +52 77732970 20; fax: +52 77732970

E-mail addresses: baier@buzon.uaem.mx (G. Baier); thermann@ sonification.de (T. Hermann).
}

Both problems can be attended when using data sonification. Sonification is the data-driven sound synthesis designed to make specific features within the data perceptible (Kramer, 1994). It has been applied to human EEG in different approaches (Hermann et al., 2002; Meinicke et al., 2004), most of which are not easily implemented for realtime applications, however. So far, the only technique that explicitly supports the auditory perception of rhythmic activity in EEG is a mixture of model-based and eventbased sonification that requires the decomposition of each EEG channel by means of a system of coupled non-linear differential equations (Baier and Hermann, 2004).

It has been argued that sonification provides an efficient way to investigate simple and complex physiologic rhythms, be they deterministic or stochastic in nature (Baier, 2001). In particular, event-based sonification, the triggering of sounds by pre-defined data features, can easily be implemented for real-time applications. It allows one to directly detect temporal relationships in the data if the 
mean period between two events is in the range where rhythms are best processed by the human ear. This range is approximately from 0.5 to $20 \mathrm{~Hz}$, i.e. it covers the $\delta, \theta$, $\alpha$, and $\beta$ band of human EEG. In a popular context, this technique was first applied to EEG data with a generalized absence seizure (for a recording see the $\mathrm{CD}$ in Baier (2001)). Some scientific examples of an event-based sonification of $3 / \mathrm{s}$ spike-wave complexes have been published recently (Baier et al., 2006). These examples demonstrate how sonification aids the characterization of complex data: recurrent wave forms (rhythms) are characterized by welldefined temporal relationships between a present event and previous events. This is expressed best in the so-called autocorrelation function, which evaluates this relationship quantitatively on a scale between -1 (completely anti-correlated) and 1 (completely correlated or identical). Rhythmic activity yields high values in the autocorrelation function, whereas non-rhythmic activity (like background activity) yields values close to zero. There is evidence that human auditory perception is based on the continuous analysis of autocorrelations in auditory data streams rather than Fourier analysis (Griffith et al., 1998). As such, it is suited to detect temporal relationships effortlessly and to combine the results to form auditory Gestalts like rhythms.

Furthermore, it was found that event-based sonification can be extended to facilitate the detection of cross-correlations, e.g. phase relationships between rhythms from different sources (Baier et al., 2005). In this case, auditory perception is especially sensitive to transient changes in spatial correlation. Taken together, these advantages suggest event-based sonification as an appropriate strategy to display multi-channel EEG recordings with their temporary changes in auto- and cross-correlations.

Epileptic activity often manifests itself as rhythms in human electroencephalogram (EEG). These rhythmic patterns are the basis for the detection of epileptic pathologies from human EEG (see e.g. Niedermeyer and Lopes da Silva, 1999). As such they are well-characterized in terms of their auto- and cross-correlations and serve as ideal data to develop, test and evaluate new sonification techniques. Here, we describe a method to generate event-based rhythms from multi-channel human EEG and present real-time sonifications of both generalized and focal epileptic activity. Among the possible applications of the method are (i) combination of the software with clinical video monitoring; (ii) combination with monitoring devices in the ICU; (iii) out-of-clinic monitoring, e.g. in patients with seizures that occur predominantly during sleep; and (iv) the combination with visual display in EEG feedback settings.

\section{Data and parameter extraction}

For the present introduction to EEG sonification, samples of several minutes length were selected from prerecorded EEGs. These included plain background, background plus artifacts, interictal focal activity, subclinical spike-and-wave runs and ictal spike-and-wave seizures.
The presented sonifications are all realized from either a 14-year-old male patient with fronto-central epileptic focus and partial seizures (denoted patient 1) or from a 12-yearold female patient with typical absence seizures of $3 \mathrm{~Hz}$ spike-and-slow-wave appearance (denoted patient 2). Clinical EEGs of patient 1 were recorded with 10/10 position of 27 scalp electrodes at a sampling rate of 500/s. Clinical EEGs of patient 2 were recorded with $10 / 20$ position of 19 scalp electrodes at a sampling rate of $256 / \mathrm{s}$. The recording equipments performed default filtering of DC offset. The data were band-pass filtered (low pass filter $15 \mathrm{~Hz}$, high pass time constant $0.1 \mathrm{~s}$ ) before entering the sound synthesis.

Data were used in a bipolar montage in the case of patient 1, and the Hjorth source derivation (Hjorth, 1980) for patient 2. The bipolar montage, while lacking a fixed reference, yields local differences of electric potentials. The Hjorth montage creates an individual reference for each electrode defined as the weighted sum of the potentials from its nearest neighbors. It thus acts (like any current source density montage) as a spatial high-pass filter that reduces spurious cross-correlations compared to common references, particularly in the case of generalized rhythms. No other manipulations of the data were performed prior to sound synthesis.

For each time series three main parameters were extracted for subsequent use (Fig. 1). First, voltage maxima were detected by means of a three-point criterium. A threepoint criterium proved sufficient for low-pass filtered data but for unfiltered data a five- or seven-point criterium is necessary to diminish the effect of high-frequency noise. Second, the voltage difference between a newly found maximum (the present maximum) and the previous minimum was calculated. Third, the time difference between the occurrence of the present and the previous maximum was memorized. In addition, for the sonification of more than one data channel, the time difference between the occurrence of a maximum in one channel and the previous maximum in another channel was evaluated.

\section{Sound synthesis}

For a sonification, the complete set of EEG data channels is fed into the working memory of the computer and

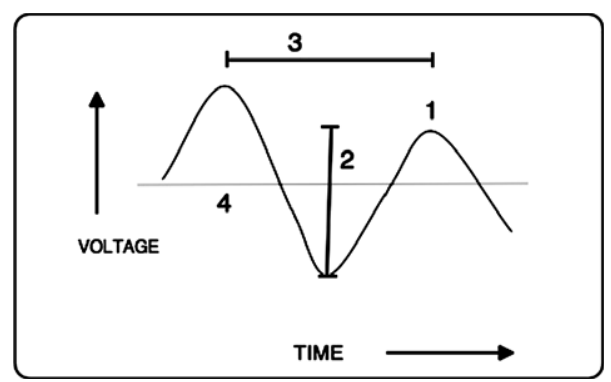

Fig. 1. Parameters for sonification. 1: voltage maximum; 2: voltage difference between present maximum and previous minimum; 3: time difference between present and previous maximum; 4: threshold voltage. 
a fixed number of channels is selected. Presently, each of the selected channels is treated equally. We describe a one-channel setting in detail; sonifications with two and three channels are also presented and sonifications of a larger number of channels can be realized by straightforward extensions of the core setting. The programs are such that they can implemented on any PC with audio card in the Linux, OS X, or Windows environment.

In epileptic EEG, the extrema of the time series (minima and maxima) are natural candidates to define events. They allow the detection of either singular events like spikes and sharp waves or repetitive (rhythmic) activity, both during seizures and in periods between seizures. Zero-crossings and inflection points are other choices but were not found to improve the result and we thus selected voltage maxima of time series as events.

Each maximum (labeled 1 in Fig. 1) triggers the playing of a sound from a pre-defined sound generator in the programming environment Supercollider 3. Supercollider is an open source package for real-time audio synthesis programming available from http://www.audiosynth.com/. The sound generator called NC consists of a Blip oscillator with constant fundamental frequency and added sinusoidal vibrato. The Blip oscillator is a band-limited impulse generator where all harmonics have equal amplitude. The generated tone is modulated with a percussive envelope Env that allows adjustment of attack time (the onset characteristics of a sound before the plateau is reached), duration of tone, and decay rate. Parameters of the Blip oscillator (amplitude, frequency, number of harmonics) and its envelope (attack, duration, decay) are controlled by parameters extracted from the time series (see Appendix A for details).

The volume level of an event in $\mathrm{dB}$ is set in a linear mapping of the voltage difference between the present maximum (that triggers the sound) and the previous minimum in a time series (labeled 2 in Fig. 1). As the range of this difference cannot be foreseen, this mapping is adjusted from the mean amplitude of the ongoing activity such that electric ongoing activity generates audible but non-intruding aural events. To avoid clipping artifacts due to the limited amplitude range of the synthesis engine, a constant maximum level is empirically adjusted for voltage differences that are larger than a given, data-dependent threshold.

The total duration of a tone, with fixed attack and decay rate, is modulated by the inter-maxima interval (labeled 3 in Fig. 1). Again the mapping was chosen linear and was kept such that a short interval leads to a short tone and a longer interval to a longer tone. Here, testing is required to assure that short tones do not lose a perceptible pitch and that long tones do not overlap strongly with the triggering of the subsequent tone.

The number of harmonics of a tone is also controlled by the inter-maxima interval as is the duration if only a single time series is sonified. This is redundant in the sense that one time series parameter controls two sound parameters. Yet it leads to a significantly improved perception of the inter-maxima interval variability by the listener. In the two-channel setting, the number of harmonics is controlled by the period between the present maximum in a given time series and the previous maximum in another time series. In both cases, we use a reciprocal mapping, i.e. a short distance leads to a high number of harmonics (a sharp tone) and a long distance results in a low number. This mapping is bound to a finite range with prefixed limits set to keep the sound within reasonable boundaries. We chose 2 as the minimum, and a data-dependent preset constant maximum number of harmonics. The fundamental frequencies of each oscillator are fixed in some cases but can be used as a sensitive sound parameter for additional clues in other cases. For the one-channel examples, $f(1)=200 \mathrm{~Hz}$, and for the two channel example of patient $2, f(1)=200 \mathrm{~Hz}$ and $f(2)=300 \mathrm{~Hz}$ were chosen to create a harmonic and non-disturbing perception for listeners with little experience with auditory data displays.

We have performed numerous experiments to use the control of the fundamental frequency (pitch) of a tone for sonification but found that by themselves neither continuous nor discrete mappings of parameters are as supportive as rhythmic displays when it comes to the detection and identification of abnormal rhythms. In fact, many realizations turned out to be either distracting (when rendered too melodic) or annoying (when rendered nonmelodic) in prolonged listening sessions. Here, we use pitch only to define the base frequency and to distinguish sounds from different data sources. However, we believe that once the rhythmic display is optimized, a carefully chosen pitch control will be an important option for the inclusion of further clues about EEG features into the sound synthesis.

In the one-channel setting, the audio channel is panned (i.e. spatially localized by linear distribution of the power between two speakers) to the middle between two speakers, and in the two-channel setting channels are panned to the left and right stereo speaker by means of the two-channel equal power panning Pan2 in Supercollider, respectively. More than two time series can be used in the Pan2 setting and we demonstrate a panning of 23 channels inspired by their position on the scalp, where the pitch allows to infer the anterior/posterior location and the stereo position indicates the hemispheric location. For multi-electrode sonifications with a demand for high spatial resolution a four or multi-channel panning is preferable.

The full program code, a step-by-step explanation how to sonify a dataset, and sound examples are provided on the following website: http://www.sonification.de/projects/ eegson/CLINPH2007/index.html

\section{Results}

\subsection{Background activity}

Fig. 2 (top) shows a recording of background activity obtained from the bipolar channel (Fz-Cz). The recording corresponds to a $20 \mathrm{~s}$ section that is free of muscle artifacts and does not contain epileptiform activity. The figure 


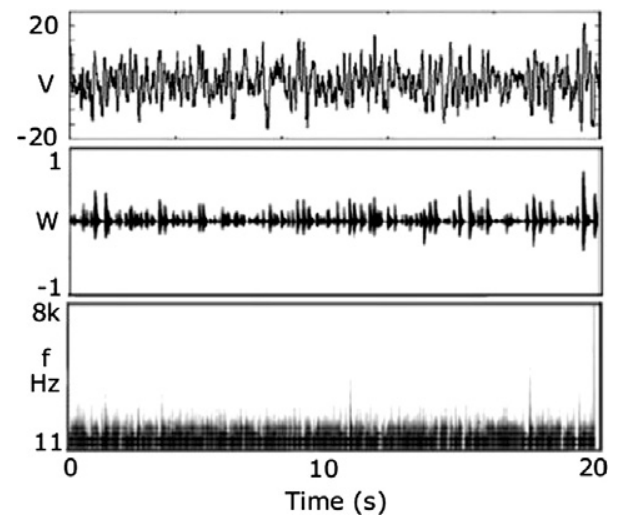

Fig. 2. (Top) Background activity from channel Fz-Cz. Linear waveform $\mathrm{W}$ (center) and spectrogram (bottom) of Sound 1: sonification of background activity in channel $(\mathrm{Fz}-\mathrm{Cz})$ in patient 1 . The $y$-axis of spectrograms is in log-scale.

displays the noisy characteristics of normal background, i.e. irregular fluctuations with oscillations at many time scales. Sound 1 (see Appendix B) is the sonification of this time series with the event-based mapping introduced above, applied in real time. Details of the mapping are given in Appendix A. For a graphical representation of the sound we included its waveform and spectrogram (Fig. 2, center and bottom). The linear wave form $\mathrm{W}$ is the computed output of the sound synthesis software and represents the signal that is sent to the speakers. The spectrogram is the running window implementation of a Fast Fourier Transform performed over overlapping wave form segments with a window size of 4096 data points.

The sound consists of continued beats at different volume levels. No regularity can be perceived in the timings of the beats, although there is a hierarchical structuring of the auditory stream due to the mixing of low, medium, and large volume events. The sound gives a representation that is consistent with the broad-band Fourier spectrum of the signal in the range selected by the band-pass filter (the $\theta, \alpha$, and lower $\beta$ band). Large amplitude muscle artifacts (that often show up in the recording at amplitudes that are more than five times larger than the mean variance of the signal shown in Fig. 2, top) create loud but not salient beats and would thus go unnoticed if they are occasional singular events. The aim of our study of epileptiform activity is to put these characteristics of the electric background into the acoustic background (silence or low volume) of the sonification.

\subsection{Activity during absence seizure}

Fig. 3 (top) shows a section of the recording of one EEG channel in patient 2 (T4 over the right temporal lobe). The section contains background activity with some muscle artifacts (at approximately 8 and $16 \mathrm{~s}$, due to body movement) and a (spontaneous) epileptic activity between 25 and $35 \mathrm{~s}$. The epileptic activity consists of repeated typical spike-wave complexes with a frequency of approximately

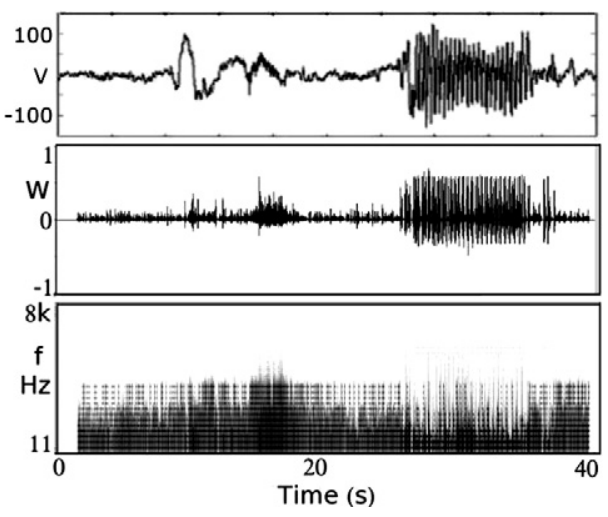

Fig. 3. (Top) Recording of channel T4 in Hjorth montage with spike and wave activity (between 25 and $35 \mathrm{~s}$ ). Waveform (center) and spectrogram (bottom) of Sound 2.

3/s and a large amplitude compared to the artifact-free background.

Sound 2 presents the sonification of the segment shown in Fig. 3 (top). Its waveform and spectrogram are also displayed (Fig. 3, center and bottom). As before, the background activity generates the almost continuous irregular sequence of tones. Most tones are of low volume due to the small differences in voltage between minimum and successive maximum. Short inter-maximum intervals lead to short durations and a comparatively large number of harmonics of most tones. The strong muscle artifact leads to notably increased volume of the tones (larger voltage differences due to the superimposed electric muscle signal), a slightly higher average number of harmonics (due to increased power near the upper end of the selected frequency range). Due to the cut-off frequency of the low-pass filter the duration of the tones does not decrease significantly during the artifact period.

During the seizure interval the amplitude mapping leads to maximum volume, the inter-maximum interval mapping leads to significantly increased durations of tones and to reduced average numbers of harmonics (c.f. the spectrogram in Fig. 3, bottom). The alternating pattern of one short and one longer tone reflects the combination of spikes (duration about $0.08 \mathrm{~s}$ ) and slower waves (duration about $0.23 \mathrm{~s}$, i.e. about three times the duration of a spike). In fact, the slow wave is followed by a small rapid oscillation, creating a third tone of low volume just before the tone corresponding to the large spike. However, this third tone can only be perceived clearly at slower sonification speed.

Sound 3 (see waveform and spectrogram Fig. 4, top and bottom) is generated as Sound 2 but this time two EEG channels were used to generate two sound event sequences that are panned to the left and right stereo channel, respectively. The two base frequencies are set to the interval of a fifth (200 and $300 \mathrm{~Hz}$, respectively). It can be heard that the spike-wave activity in the two channels is strongly correlated but displays similar rhythmic properties. Outstanding are sharp tones in channel F4 (right stereo channel) that 


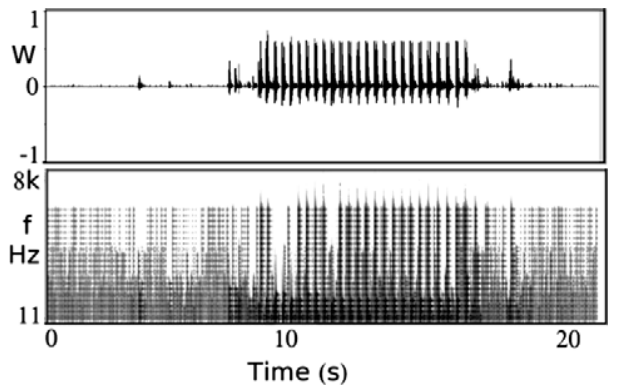

Fig. 4. Waveform (top) and spectrogram (bottom) of one audio channel of Sound 3: background and absence seizure in patient 2, channels T4 (left) and F4 (right) in stereo panning starting from second 20 in Fig. 3, top. Oscillator frequency is $200 \mathrm{~Hz}$ for channel T4, and $300 \mathrm{~Hz}$ for channel F4.

occur some time after the seizure initiates. These possess high volume, long duration, and a large number of harmonics. They can be attributed to large spikes in channel F4 that are preceded by a large inter-maxima interval in F4 and by a very small inter-maxima interval due to a small spike in channel T4. (This small spike in channel T4 is what caused the before-mentioned "third tone" of channel T4 in Sound 2.)

The two-channel display makes it audible how the seizure at its onset requires some time before the absence rhythm is fully established. This can be discerned as a gap in the spectrogram of the seizure activity after the two initial strong beats in both channels. Similarly at the end, although the loss of amplitude is sharp (see Fig. 3, top), there is a transient phase of rhythmic decomposition before the previously exhibited background activity is resumed.

In preliminary listening tests, subjects were able to correctly identify short subclinical trains of $3 / \mathrm{s} \mathrm{spike} \mathrm{and} \mathrm{wave}$ complexes in EEGs from other patients after a single training session with sounds generated from patient 2 .

\subsection{Interictal activity}

Fig. 5 (top, lower trace) shows a recording of the EEG of patient 1 during the interictal period. In that period, the dynamics is dominated by background activity as in Fig. 2 (top). However, occasionally, there are pathologic transients associated with the presence of an epileptic focus. In patient 1 the focus is located such that its activity can be picked up best by electrode configurations ( $\mathrm{Fz}-\mathrm{Cz})$ and (Cz-Pz). As shown in Fig. 5 (top, lower trace), the focal activity is transient, normally no longer than $1 \mathrm{~s}$ ) and has a clear rhythmic component with a frequency of about $10 \mathrm{~Hz}$, i.e. in the $\alpha$ band. The rhythm appears to be amplitude modulated but the actual shape of this modulation varies strongly from instance to instance.

Sound 4 is produced with the settings used in Sound 1 . The waveform and spectrogram in Fig. 5 (center and bottom) demonstrate that this mapping is successful in separating the focal rhythm from the background. Listening to the sound, the impression of a change in the rhythm

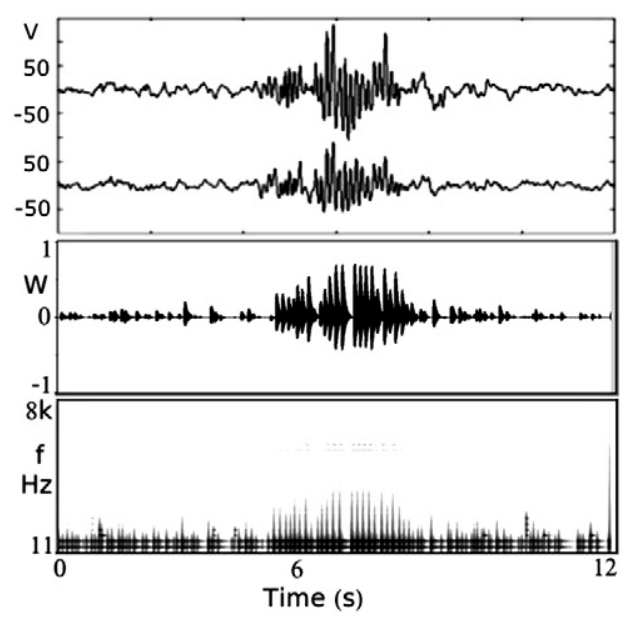

Fig. 5. (Top) Focal rhythmic activity in the recording of channel (Fz-Cz), lower trace; and combination $(\mathrm{Fz}-\mathrm{Cz})-(\mathrm{Cz}-\mathrm{Pz})$, upper trace, with improved rhythm/background ratio. Waveform (center) and spectrogram (bottom) of Sound 4.

(e.g. as expressed in the variation of inter-maxima intervals) is confirmed by the increased volume and thus the focal rhythm is easy to identify. It should be noted that in this patient the healthy occipital $\alpha$ rhythm (which appears preferentially in electrodes $\mathrm{O} 1$ and $\mathrm{O} 2$ with eyes closed) is not manifested in the electrodes used in Fig. 5. Nevertheless, the continuous presence of tones due to background activity calls for further improvement of the mapping, particularly in cases where the focal activity is of small amplitude.

From inspection of the EEG it is evident that channels $(\mathrm{Fz}-\mathrm{Cz})$ and $(\mathrm{Cz}-\mathrm{Pz})$ pick up the same rhythm and that they are strongly anti-correlated. Because this is not the case for normal background activity we use the combination (Fz$\mathrm{Cz})-(\mathrm{Cz}-\mathrm{Pz})$ as an input for the sound synthesis to improve the variance of the rhythm as compared to the mean variance of the background in the vicinity. This is shown in Fig. 5 (top, upper trace). As a result the focal rhythm becomes dominant in both visual (Fig. 6, top) and audio displays. Sound 5 is a sonification of low-amplitude focal activity from data of $(\mathrm{Fz}-\mathrm{Cz})-(\mathrm{Cz}-\mathrm{Pz})$. The increase in volume particularly aids the detection of the rhythm in the second, comparatively shorter occurrence (see waveform and spectrogram Fig. 6, center and bottom).

In both, Sounds 4 and 5, the applied linear mapping of the number of harmonics does not seem to contribute strongly to the separation of rhythm from background (as it does e.g. in Sounds 2 and 3). This is because the background activity also has significant power in the $\alpha$ band and the focal activity is not as unusual as e.g. the $3 / \mathrm{s} \mathrm{rhythm} \mathrm{of}$ an absence seizure. This is also reflected in the comparatively weak discrimination of rhythm from background in the spectrogram of Fig. 6, bottom. As such the number of harmonics mapping can still be considered a free parameter for the present sonification of focal activity.

For Sounds 6 and 7 the mapping was slightly modified. Inspection of the EEG of patient 1 shows that the focal 


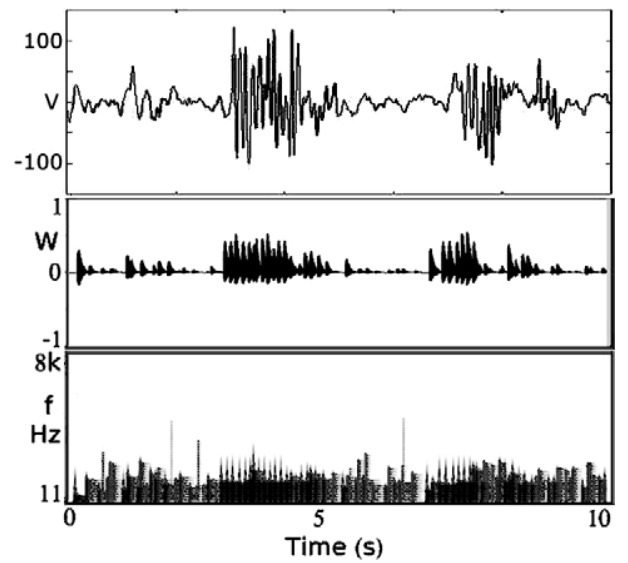

Fig. 6. (Top) Two consecutive transients of focal rhythmic activity in the combination $(\mathrm{Fz}-\mathrm{Cz})-(\mathrm{Cz}-\mathrm{Pz})$ with improved rhythm/background ratio. Waveform (center) and spectrogram (bottom) of Sound 5. Data displayed in Fig. 5, top, lower trace.

activity has a stable location and a preferred frequency between 8 and $12 \mathrm{~Hz}$. We can thus adjust the mapping of the number of harmonics in accordance with this finding. A negative quadratic mapping according to the function $y(t)=$ const $* t-t^{2}$ was chosen with a maximum at $t=0.1 \mathrm{~s}$ corresponding to $10 \mathrm{~Hz}$. In addition, a finite amplitude threshold was chosen to suppress all sounds below a given minimum volume. This further reduces the (insignificant) background contributions. Sound 6 (see waveform and spectrogram Fig. 7, top and bottom) is the sonification of a long continuous segment of EEG with this modified mapping. Both waveform and spectrogram indicate the successful suppression of background activity and thus an improved identification of segments with focal rhythm (compare the spectrograms Fig. 6, bottom and 7, bottom).

Sound 7 was created similar to Sound 6, except that three data channels were panned in a stereo setting (see waveform and spectrogram Fig. 8, top and bottom). The middle channel is identical to the one used in Sound 6 . The left and right channel are obtained from combination of two correlated channels that also display focal $\alpha$ activity, albeit at lower amplitudes. Seven segments with focal rhythm of different length and intensity were chosen to demonstrate both the variability and the stability of the

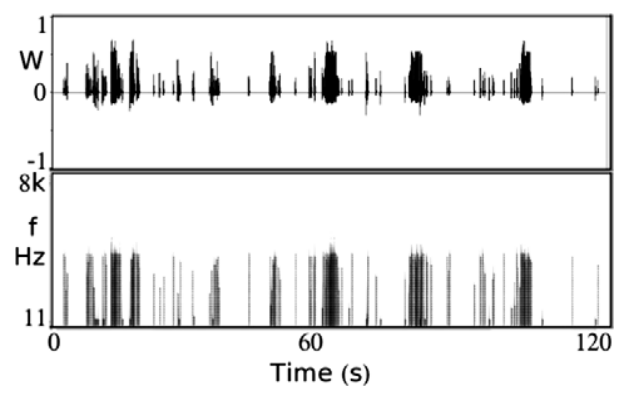

Fig. 7. Waveform and spectrogram of Sound 6: transient focal activity in patient 1 with improved rhythm/background ratio in $(\mathrm{Fz}-\mathrm{Cz})-(\mathrm{Cz}-\mathrm{Pz})$. Sonification of a longer segment with various transients with data similar to those in Fig. 6 (top).

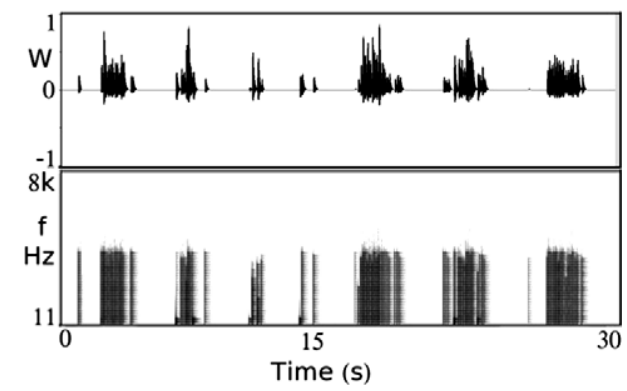

Fig. 8. Waveform and spectrogram of Sound 7: seven glued together segments of transient $\alpha$ activity in patient 1 (data similar to those in Fig. 6, top). Improved rhythm/background ratio and quadratic mapping of the number of harmonics. Sonification as in Sound 6 but with three channel stereo display: (Fz-Cz)-(Cz-Pz) (middle); (C3-F3)+(C4-F4) (right); $-(\mathrm{P} 3-\mathrm{O} 1)-(\mathrm{P} 4-\mathrm{O} 2)$ (left). Only the signal of the left stereo channel is shown.

phenomenon. The stereo display gives a spatial impression of the spreading of the focal rhythm: small amplitudes are perceived as centered (the activity is restricted to the middle channel) but at larger amplitudes a spatial spreading of the activity (with dominance to the right channel from posterior electrodes) can be perceived.

In preliminary listening tests subjects were able to identify interictal focal activity in the EEG of patient 1 with only one training session. They were also able to roughly estimate the average number of occurrences, the duration of individual events and the intensity of the rhythm, the latter after listening to examples of the relationship between EEG amplitude and sound level.

\subsection{Transition to epileptic seizure}

The multi-channel display can be extended and we present a sonification that comprises 19 channels in a stereo display. To set-up this display we first defined Cartesian coordinates for each electrode in the plane. The $X$ axis is defined by the left-right extension on the scalp (ear to ear) and the $Y$ axis by the posterior-anterior line (neck to nose). Then we used the $X$ coordinate of a given electrode to control its stereo panning. As the large number of channels does not permit unambiguous identification of electrodes we added pitch information as an additional clue about the $Y$ coordinate of an electrode. The most posterior electrodes (O1 and $\mathrm{O} 2)$ were assigned a base pitch and the $Y$ coordinate was then mapped linearly to pitch leading to highest pitch for the prefrontal electrodes.

Sound 8 demonstrates the transition from background activity to a clinical epileptic seizure in patient 1 (full EEG recording in Fig. 9, see waveform and spectrogram Fig. 10, top and bottom). It starts with background activity that lasts for about $3 \mathrm{~s}$ and then quickly develops activity in the $\alpha$ band that is distributed over more than half of the channels (central section of the figure). The $\alpha$ activity then disappears and for about half a second there is no notable rhythm in the EEG (depression). The clinical seizure then 


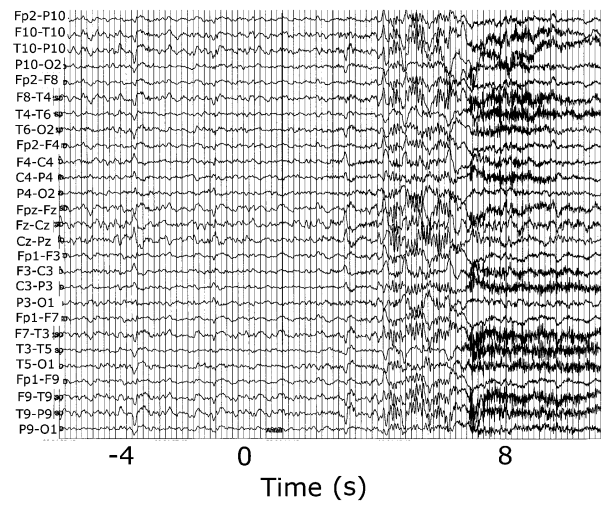

Fig. 9. EEG of transition to seizure in patient 1. Bipolar montage.

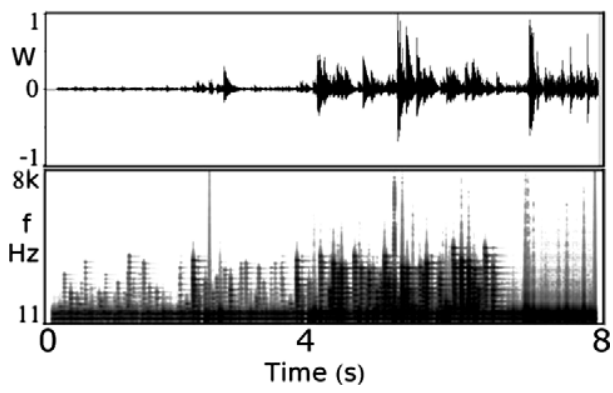

Fig. 10. Waveform and spectrogram of Sound 8. Data shown in Fig. 9. Sonification as in Sound 7 but with 19 channel stereo display. Only the signal of the right stereo channel is shown. The clinical seizure starts at the rightmost strong outburst in the waveform and the recording was interrupted about $2 \mathrm{~s}$ into the seizure. See text for details.

starts with muscle activity which is superimposed on brain activity and dominates it for most of the seizure period. As can be seen in the waveform and the spectrum, the chosen mapping highlights the transient ictal activity in both volume and brightness. During this transient there is a dominance of the right over the left hemisphere in terms of the volume of the rhythm. This is not obvious from the original voltage recordings or voltage mappings. Comparing the rhythmic activity in the $\alpha$ band prior to the fully developed seizure rhythm (seconds 4-6 of Sound 8) with the interictal focal activity (Sound 7), we find that there are characteristic differences in their distribution (interictal activity is restricted to fewer electrodes) and their rhythmic pattern. In preliminary listening tests these two rhythms (when presented in isolation) were therefore generally labeled as "different" by most subjects.

\section{Discussion}

The sonification of human EEG is a new field of investigation. For our present study we have chosen recordings with two types of rhythmic transients that are typical of epileptic dynamics. The frequency of most epileptic pathologies in the EEG falls into the range $0.5-20 \mathrm{~Hz}$ (see e.g. Gibbs and Gibbs, 1952) and is therefore ideally suited to be sonified in an event-based way for real-time applications. In a previous approach, the EEG signal was decom- posed into a large number of narrow-band filtered signals that were then transformed into sequences of excitations of equal amplitude to obtain a spectro-temporal representation of it (Baier and Hermann, 2004). However, that technique requires the simultaneous integration of a large number of differential equations and an averaging procedure to yield significant results and, in its conventional implementation, is not suited for on-line analysis of the EEG. See, however (Chernihovskyi et al., 2005), for a possible real-time implementation. The present technique is much simpler in that it extracts the rhythmic properties directly from extrema of the EEG recording.

Event-based sonification of band-pass filtered time series was used for auditory EEG feedback by Hinterberger and Baier (Hinterberger and Baier, 2005). They created a multiinstrument set-up for the simultaneous listening of all frequency bands from 0.1 to $40 \mathrm{~Hz}$. This allows an auditory representation of the whole signal complexity, in particular of background activity, but does not specifically support the perception of transient pathologic features which is the focus of interest in clinical monitoring. For monitoring purposes it is essential for the sonification to separate background from pathologic features as was achieved in the examples above.

To simplify the detection of rhythmic events in this frequency range, a low-pass filtering is required in addition to the standard high-pass filter of most recording equipments. The low-pass filter can be included directly in Supercollider and serves to get rid of high-frequency background components and to reduce the influence of muscle artifacts which contribute broad-band noise to the EEG.

As with visual techniques, we exploited the amplitudes of epileptic pathologic features to enhance their representation. The mapping of amplitude to volume is a natural way to let rhythmic events stand out and is particularly supported by various types of epileptic rhythms that show a clear amplitude pattern in the EEG. The increase in amplitude (as in Fig. 3, top) is mainly due to a process of cortical (hyper)synchrony and helps to distinguish an abnormal rhythm from the multiple lower amplitude rhythms that form the background activity.

The choice to use the inter-maxima interval to control two different sound parameters was based on the experience that this data feature may serve two different purposes. First, the control of tone duration leads to an improved perception of rhythm, independent of its frequency. A duration that is adequate for a slow rhythm of $1 / \mathrm{s}$ will be too long to allow perception of the individual beats of a 15/s rhythm. And vice versa, a short duration that allows the perception of a $15 / \mathrm{s}$ event as a rhythm (and not as a continuous sound) will lead to the impression of a $1 / \mathrm{s}$ rhythm as a series of isolated events. The mapping of inter-maxima intervals to duration solves this problem naturally with a simple data feature. Second, mapping of inter-maxima intervals to the number of harmonics of a tone modulates its brightness. Brightness is a sound parameter that is sensitively perceived by the human ear and is 
used customarily as a cue for information about the sound sources. In data sonification this can be exploited if the mapping is in the appropriate range, where changes in the number of harmonics are most easily perceived. The sonifications of both background and epileptic rhythms give a clear indication of the distribution of inter-maxima intervals. Compare for example the background in Sounds 1 and 2. As the background is overwhelmingly irregular, any change to a more regular sequence of inter-maxima intervals is readily perceived not only by the more regular triggering of events but also by the clearer structuring of the distribution of harmonics.

A most interesting property of sound perception that has not yet been used explicitly in the sonification of human EEG is that it conveys information about space. Sonifications 3 and 7 map the sounds triggered by different data channels to different positions in space. This allows an easy identification of sound sources even if their fundamental frequencies are identical as in Sound 7. If two rhythms from separate sources are intertwined and display a nontrivial relationship as in Sound 3, an additional difference in fundamental frequency aids the resolution of individual contributions to the perceived whole of a sound stream. From the present examples we infer that spatial separation of sound sources can be used in the future to further refine the sonification for clinical purposes. If combined with an inter-maxima interval mapping between channels (as in Sound 3) this will particularly facilitate the perception of changes in cross-correlations (Baier et al., 2005) and might then be used to analyze signals with more subtle rhythmic changes (like the correlation changes that precede epileptic seizures (Mormann et al., 2003)).

The combination of panning and pitch in the 19-channel display of Sound 8 (Fig. 10, center and bottom) provides a simple acoustic mapping of the complete EEG. This allows for the monitoring of groups of channels participating in a series of events as for example the pre-seizure activity in the $\alpha$ band. Some training is required to interpret the complex auditory streams in terms of participating electrodes but test listeners found the left-right stereo display of the left-right electrode position intuitive to understand and the low-high pitch mapping of the back-to-front position easy to learn. Nevertheless a display with more than two channels will be helpful for tasks where the observation of activity from individual electrodes is monitored in the presence of all other signals.

In preliminary listening tests, we found the present sonifications in the stereo setting to be effective in terms of recognition of previously learned patterns. However, systematic listening tests are required that specifically test for (i) listener's ability to recognize a given event in EEGs of the same and of different patients; (ii) the capability to differentiate a given pattern from artifacts and other rhythmic events in the auditory display only; and (iii) the comparison of results with a visual display only, an auditory display only, and a combined audio-visual display to evaluate the performance across the two modes of perception.
Epilepsy-related EEG rhythms in different patients may have strongly varying features, like spike or wave periods, duration of a wave trains, composition, location or distribution on the scalp, and others (for an overview see chapter 27 in Niedermeyer and Lopes da Silva (1999)). This means that a single set of sound synthesis parameters from one patient will most likely not be optimal for other patients. This is especially the case if a patient-specific optimization is done as in the case of patient 1 for Sounds 6 and 7. Even for a second patient with the same type of diagnosed epilepsy, conditions may vary, for instance, the location of the focus will probably be different and the focal activity may therefore appear at different electrode positions on the scalp; and the fundamental frequency of interictal focal activity may differ. Thus, one would have to switch to different data channels for the sonification and would have to adjust the filter properties for the number of harmonics parameter in order to get optimal results. Rather than to perform these changes off-line, we propose that this can best be done interactively. Interactivity was identified to be a key feature of sonifications in real-world applications (Hermann and Hunt, 2005) and the aural monitoring of human EEG provides a good example of this. The presented implementation with Supercollider is well suited for this extension. The selected data channels and other sonification parameters (e.g. the center frequency of the filter used to control the number of harmonics) are simply declared variables of a graphical user interface that accompanies the sound synthesis. This interface then allows on-line selection and adjustment of parameters for optimal performance.

A problem with EEG data that has not yet been dealt with explicitly is the separation of electric brain activity from muscle artifacts. From listening experience we find that one can learn to recognize artifacts quite easily because, just like epileptic rhythms, they lead to characteristic sound objects (c.f. the artifact in Sound 2, Figs. 4 and 5). Thus, in analogy with routine visual inspection, by listening to long sections of sonified EEG one comes to establish an (internal) classification of the auditory objects from artifacts. Nevertheless, it would be helpful to implement automatic artifact detectors that help to suppress sound synthesis from unambiguous artifacts. In fact, it is by listening to sonified artifacts that one identifies cues about the features that help classify them. Apart from their large amplitudes we find their high-frequency rhythms, as represented for instance by their intermaxima interval distributions, a simple feature that helps distinguish them from most epileptic brain activity. Pre-processing the data accordingly would be no hindrance for the sonification as in clinical monitoring a delay of a few hundred milliseconds is not critical.

Finally, if the proposed sonification is implemented in an EEG feedback environment like POSER (Hinterberger and Baier, 2005) it will become possible to use it for therapeutic training of epileptic patients that so far is restricted to visual feedback, see e.g. Elbert et al. (1984), and Birbaumer et al. (1991). More recently combined audio-visual feedback has 
been applied in an EEG feedback study of slow cortical potentials (Hinterberger et al., 2004) and rhythms in the $\beta$ range (Egner and Gruzelier, 2004) but the audio part of the used programs was restricted to rather simple beeps. As argued in Hinterberger and Baier (2005), an improved sonification based on multiple data parameters may also lead to more advanced interactions with the brain potentials.

To conclude, event-based sonification of EEG allows quick detection and identification of specific rhythmic events without need to continuously watch a visual display. Single or multi-channel settings are available and parameters can be fine-tuned for specific rhythms, e.g. in epilepsy. The presented algorithms use few data parameters for a robust display that can easily be implemented for on-line sound synthesis in a clinical or non-clinical environment.

\section{Acknowledgments}

We thank Drs. H. Muhle and G. Wiegand, Clinic for Neuropediatry, Kiel, for data acquisition, selection and interpretation. G.B. acknowledges support from CONACyT Mexico (Project No. 40885-F) and from the Klaus Tschira Foundation, Heidelberg. G.B. thanks U. Kummer and M. Müller for support and discussion.

\section{Appendix A. Sound synthesis}

Sound generation and parameter control is realized with Supercollider 3, an open source package for real-time audio synthesis programming available from http:// www.audiosynth.com.

\section{Synthesizer definition}

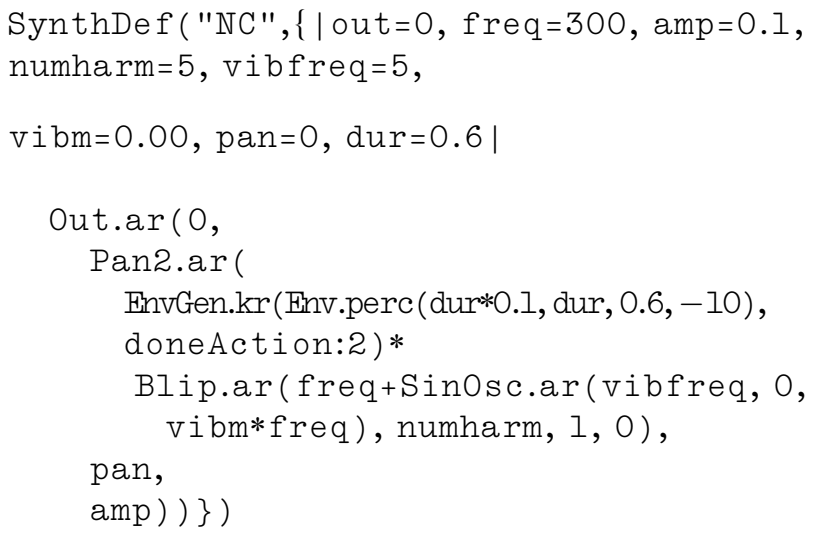

\section{Mappings}

- Parameter dur (duration of tone): for $0.1<$ dur $<0.5$ : dur $=\mathrm{mp} 1 *($ PresentMaxTime-PreviousMaxTime $)$.

- Parameter numharm (number of harmonics) for one channel: for $1<$ numharm <21: numharm $=2+(\mathrm{mp} 2 /$ (PresentMaxTime-PreviousMaxTime1)).
- Parameter numharm (number of harmonics) for two channels 1 and 2: for $1<$ numharm <21: numharm = $2+(\mathrm{mp} 2 /($ PresentMaxTime1-PreviousMaxTime $)$ )); (and vice versa for the second channel).

- Parameter numharm (number of harmonics) for alpha activity; for $1<$ numharm < 31: numharm $=\mathrm{mp} 2 *(100 *$ (StepNumber-PreviousTimeMax)-((StepNumber-PreviousTimeMax)*(StepNumber-PreviousTimeMax))).

- Sounds 6 to 8 :numharm $=(\mathrm{mp} 2 *(100 *($ StepNumberPreviousTimeMax) - ((StepNumber-PreviousTimeMax)* (StepNumber-PreviousTimeMax)))).round.

- Parameter amp (amplitude): for $0<a m p<1$ : amp $=$ $\mathrm{mp} 3 *($ PresentMaxVolt-PreviousMinVolt)).

- Sounds 6 to 8: Mapping of parameter amp applied for $0.3<\operatorname{amp}<1$.

\section{Appendix B. Supplementary data}

Sound files and supplementary data associated with this article can be found, in the online version, at doi:10.1016/ j.clinph.2007.01.025 and http://www.sonification.de/projects/ eegson/CLINPH2007/index.html.

\section{References}

Baier G, Hermann T. The sonification of rhythms in human electroencephalogram. In: Proceedings of the 10th International Conference on Aufitory Display, Sydney, July 6-9, 2004.

Baier G, Hermann T, Lara O, Müller M. Using sonification to detect weak cross-correlations in coupled excitable systems. In: Proceedings of 11th International Conference on Auditory Display, Limerick, July 6-9, 2005.

Baier G, Hermann T, Stephani U. Sonified epileptic rhythms. In: Proceedings of 12th International Conference on Auditory Display, London, June 20-23, 2006, p. f35.

Baier G. Rhythmus (in German). Reinbek bei Hamburg: Rowohlt Verlag; 2001.

Birbaumer N, Elbert T, Rockstroh B, Daum I, Wolf P, Canavan A. Clinical-psychological treatment of epileptic seizures: a controlled study. In: Ehlers A, editor. Perspectives and Promises in Clinical Psychology. New York: Plenum; 1991. p. 81-96.

Chernihovskyi A, Mormann F, Müller M, Elger C, Baier G, Lehnertz K. EEG analysis with nonlinear excitable media. J Clin Neurophysiol 2005;22:314.

Egner T, Gruzelier J. EEG biofeedback of low beta band components: frequency-specific effects on variables of attention and event-related brain potentials. Clin Neurophysiol 2004;115:131.

Elbert T, Rockstroh B, Lutzenberger W, Birbaumer N, editors. Selfregulation of the Brain and Behavior. New York: Springer; 1984.

Gibbs F, Gibbs E. Atlas of Electroencephalography, vol. 2. Reading: Wesley; 1952.

Griffith T, Büchel C, Frackowiak R, Patterson R. Analysis of the temporal structure in sound by the human brain. Nat Neurosci 1998;1(5):422.

Hermann T, Hunt A. An introduction to interactive sonification. IEEE Multimedia 2005;12:20.

Hermann T, Meinicke P, Bekel H, Ritter H, Muller H, Weiss S. Sonifications for EEG data analysis. In: Proceedings of the 8th International Conference on Auditory Display, Kyoto, 2002, p. 37-41.

Hinterberger T, Baier G. Parametric orchestral sonification of EEG in real-time for the self-regulation of brain states. IEEE Multimedia 2005; $12: 70$. 
Hinterberger T, Neumann N, Pham M, Kübler A, Grether A, Hofmayer $\mathrm{N}$, et al. Brain-based feedback and communication system. Exp Brain Res 2004;154:521.

Hjorth B. Source derivation simplifies topographical EEG interpretation. Am J EEG Technol 1980;20:121.

Kramer G, editor. Auditory Display - Sonification, Audification, and Auditory Interfaces. Addison-Wesley; 1994.
Meinicke P, Hermann T, Bekel H, Müller HM, Weiss S, Ritter H. Identification of discriminative features in EEG. J Intell Data Anal 2004;8:97-107.

Mormann F, Kreuz T, Andrzejak R, Rieke C, David P, Lehnertz K, et al. Epileptic seizures are preceded by a decrease in synchronization. Epilepsy Res 2003;53:173.

Niedermeyer E, Lopes da Silva F, editors. Electroencephalography. Philadelphia: Lippincott Williams and Wilkins; 1999. 